$16^{\text {th }}$ International Congress of Metrology, 12008 (2013)

DOI: $10.1051 /$ metrology/201312008

C) Owned by the authors, published by EDP Sciences, 2013

\title{
2013 L'année des premiers diplômés en métrologie en Algérie à l'ère du LMD
}

\author{
Nafissa. Khennafi-Benghalem \\ Unité de recherche des matériaux émergents \\ Institut d'optique et de mécanique de précision \\ Université Ferhat Abbas Sétif 1 Algérie
}

\begin{abstract}
Abstrait. 2013 est l'année de la première promotion d'étudiants métrologues reconnus au grade de Licence à l'ère du LMD par l'institut d'optique et mécanique de précision de l'université Ferhat Abbas Sétifl Algérie. Les premiers diplômés de l'IOMP ont intégré le programme en septembre 2010, et sont en juin 2013 titulaires d'une Licence professionnelle en métrologie. Ces trois années d'études ont été un chemin semé de connaissances et d'acquisitions pour cette promotion qui a poursuivit des études bien denses en la matière par l'ensemble de la profession. Cette étape de formation a également permis à chacun de retrouver une confiance et une assurance dans ses capacités d'apprentissage, d'acquérir, d'approfondir et de diversifier ses connaissances dans des disciplines fondamentales ouvrant sur un grand secteur d'activité, d'acquérir des méthodes de travail et de se sensibiliser à la recherche.
\end{abstract}

\section{Introduction}

Parmi les reproches souvent adressés à l'ancien système de l'enseignement supérieur se trouve celui qui souligne que l'université assurait des formations académiques qui étaient loin de concorder avec les attentes des secteurs utilisateurs. Dans le LMD : il est question d'assurer des formations dites «professionnalisantes », c'est-à-dire en phase et en adéquation avec les besoins spécifiques des différents partenaires. L'explosion du nombre d'étudiants est un des signes les plus visibles du développement de l'enseignement supérieur à l'échelle mondiale [1-3]. L'émergence de nouveaux besoins socioéconomiques, la concurrence entre les établissements et la place importante prise par les universités américaines et asiatiques, posent la réforme des universités comme nécessité [4] dans l'institut d'optique et de mécanique de précision, la mise en œuvre du schéma de la réforme dite LMD, a bien démarré en 2005 dans le cadre d'une carte universitaire des offres de formation [5]. Dans le souci d'établir un équilibre entre la vocation de l'institut en optique et en mécanique de précision, il a été jugé que cette relation ne peut être réalisée si on n'intègre pas des formations en métrologie [6] pour élever les niveaux de savoirs, créer et diversifier les dimensions professionnalisantes qui donnent de la lisibilité et des repères aux certifications, dans une démarche d'insertion professionnelle qui ne se borne pas à l'occupation d'un poste de travail [7].

\section{L'application du LMD et l'insertion des formations en métrologie à l' institut d'optique et de mécanique de précision}

Depuis 2005, année de la première application du système LMD à l'institut d'optique et de mécanique de précision, les enseignants n'ont cessé de créer dans le cadre de la généralisation de ce système de nouvelles formations relatives au titre de l'institut des parcours en Licence dans 5 domaines de formation. Le début s'est fut assez timide ou on a ouvert des formations professionnelles en optométrie, par la suite les formations se sont multipliées et la formation académique n'a été retenue qu'en 2007. A côté de ces nouvelles formations, subsistent encore les diplômes nationaux ancien régime (Magister, Doctorat...) en attente d'une intégration complète dans le système LMD et les diplômes

Auteur correspondant : n_benghalem@hotmail.com 
d'université proposés au titre de la formation initiale et de la formation permanente.

La réforme LMD a supprimé progressivement l'organisation des deux cycles de graduation de l'enseignement supérieur universitaire. Un premier cycle de trois ans, sanctionné par un diplôme universitaire d'études Appliquées DUEA, est consacré à la formation technique et appliquée. Le deuxième cycle est consacré à la formation fondamentale approfondie et est soit un cycle long de cinq ans, sanctionné par un diplôme d'ingénieur d'état, l'organisation de la post-graduation en magister n'a pas été elle aussi épargnée, d'après les informations recueillies la dernière promotion en DEUA a été organisée en septembre 2011 et celle d'ingénieur en juin 2012. Les formateurs ont buché pour valoriser la formation en métrologie et contrôle industriel en la sanctionnant par un second diplôme en master2 métrologie qui sera actif en septembre 2013. Cette mesure permet la création d'une discipline universitaire en métrologie et la possibilité de poursuite d'études plus poussées pour ces étudiants : qui sera à la hauteur de la reconnaissance attendue pour notre formation et notre profession.

\section{Formation à L'IOMP}

L'institut d'optique conjugue une formation en optique expérimentale et une formation en mécanique de précision. Il a comme ambition de former des cadres techniques et des chercheurs alliant de solides bases théoriques dans le domaine de l'optique géométrique et non linéaire à des connaissances approfondies dans les techniques de la mécanique de précision, d'élaboration et de caractérisation des matériaux. Le tableau 1 nous présente l'offre de formation de L'IOMP en LMD en licence de 2005 à 2013 et en master 2008-2013. Il nous permet d'avoir une information précise sur la nouvelle organisation des études et les diplômes proposés par L'IOMP

Tableau 1 : Liste de formation en LMD à L'IOMP

\begin{tabular}{|l|l|l|}
\hline Licence & \multicolumn{1}{|c|}{ Intitulés de la formation } & \multicolumn{1}{|c|}{$\begin{array}{c}\text { Responsable de } \\
\text { l'équipe de } \\
\text { spécialité }\end{array}$} \\
\hline L(MA) & Mécanique Appliquée & Pr.Zegadi Rabah \\
\hline L(OIP) & $\begin{array}{l}\text { Optique Instrumentale et } \\
\text { Photonique }\end{array}$ & Dr. Manallah Aïssa \\
\hline L(TM) & Technologie des matériaux & Dr. Kolli Mostafa \\
\hline L(MCI) & $\begin{array}{l}\text { Métrologie et contrôle } \\
\text { industriel }\end{array}$ & $\begin{array}{l}\text { Pr. Bouamama } \\
\text { Larbi }\end{array}$ \\
\hline L(O) & Optométrie & Pr. Bouzid Djamel \\
\hline $\mathrm{M}(\mathrm{MM})$ & Mécanique des matériaux & $\begin{array}{l}\text { Pr. Hamidouche } \\
\text { Mohamed }\end{array}$ \\
\hline $\mathrm{M}(\mathrm{MF})$ & Mécanique Fine & Dr. Roumili Fouad \\
\hline $\mathrm{M}(\mathrm{OPA})$ & $\begin{array}{l}\text { Optique et photonique } \\
\text { Appliquées }\end{array}$ & Dr.Meguellati Saïd \\
\hline $\mathrm{M}(\mathrm{M})$ & Métrologie & $\begin{array}{l}\text { Dr. Benghalem } \\
\text { Nafissa }\end{array}$ \\
\hline \hline
\end{tabular}

\section{Domaines, filières et spécialités}

Actuellement les cinq Licences et les quatre Masters assurés par l'IOMP dont les intitulées ont été indiquées respectivement dans le tableau 1 se répartissent en un seul domaine de sciences et technologies, et une seule filière optique et mécanique de précision toutes les formations sont de nature académique à l'exception de la spécialité métrologie et optométrie qui sont de nature professionnelle

\subsection{Formation spécialisée en métrologie en LMD}

\section{21 Licence métrologie et contrôle industriel}

Dans le domaine des sciences techniques et dans la filière optique et mécanique de précision une formation en métrologie et contrôle industriel est offerte aux étudiants ayant acquis les deux premiers semestres. Une promotion à peu prés de vingt étudiants est inscrite chaque année depuis septembre 2011 pour poursuivre des formations dans le domaine, cette formation est assez appréciée par les étudiants et on remarque aucun départ ou aucune annulation d'inscription.

\subsubsection{Première promotion des étudiants inscrits en Licence métrologie et contrôle industriel (2010- 2013)}

C'est une promotion composée de dix neuf étudiants autonomes et motivés, ayant le goût d'entreprendre, et qui ont un fort intérêt pour les applications concrètes et le travail pratique. Leur choix de cette licence professionnelle est justifié par leur engagement dans la vie active sur un sujet porteur, en se formant à l'université tout en bénéficiant de visite et de stage pratique en entreprise. Au cours de l'année universitaire, les étudiants suivent des enseignements à l'institut où ils acquièrent des connaissances pratiques, méthodologiques et théoriques, ils réalisent au cours du 6eme semestre un mini projet organisé autour d'une problématique s'appuyant sur une réalité puisée dans le domaine de la métrologie ou industriel de référence, pour une durée totale d'environ 5 mois et complètent leur formation en préparant une soutenance du travail accompli devant un jury spécialisé.

\subsection{Moyens pédagogiques et techniques de la formation en métrologie}

Les programmes s'organisent dans un parfait équilibre entre théorie et travaux pratiques spécifiques dirigés et encadrés par les enseignants. Tout le contenu de la formation du futur métrologue (la théorie, les techniques, la méthodologie, l'approche pédagogique) est enseigné dans un esprit de recherche et d'expérimentation. Cette pédagogie active, guidée par le plaisir d'apprendre et d'expérimenter, permet une véritable intégration de ce qu'est la métrologie. L'équipe pédagogique veille au 
respect des programmes et reste à l'écoute des étudiants pour leur apporter l'aide et les conseils nécessaires à une orientation judicieuse. Les stages en entreprises font partie intégrante de la formation. Un certain nombre de moyens sont mis à la disposition de la pédagogie et des étudiants; il s'agit notamment de :

- des salles de cours

- des salles de TP

- des salles informatiques (tous les postes disposent d'un accès à Internet).

L'institut dispose aussi de six laboratoires pédagogiques: de métrologie, d'électricité, de matériaux, d'usinage du verre, d'optique et de conception, dotés respectivement de dispositifs relatifs à la vocation correspondante, on cite des instruments et des appareils de mesure dimensionnelle et électrique, d'analyse, des moyens de fabrication des composants optiques, des instruments optiques et de conception qui sont accessibles aux étudiants. De plus l'institut dispose d'un Hall de technologie : Les étudiants des formations métrologiques y réalisent des travaux pratiques sur des machines comparables à celles utilisées en industrie. On signale l'existence d'une bibliothèque riche d'une collection conséquente de quelques centaines d'ouvrages scientifiques et techniques et de culture générale, la bibliothèque permet aux étudiants de se familiariser avec l'univers technologique et d'enrichir leurs travaux. Un large choix de revues est également à leur disposition, de même que les travaux et rapports des promotions précédentes, qui peuvent y être consultés.

\subsection{Ventilation des mini-projets pour les étudiants : $3^{\text {ème }}$ année métrologie et contrôle industriel}

L'encadrement des mini projets est assuré par une équipe du domaine qui propose des thèmes comme c'est déjà mentionné dans le§3.2. Les thèmes traités pour ce présent semestre sont illustrés dans le tableau 2 et par la suite un résumé explicatif est exposé pour plus de compréhension.

\section{Remarque}

$>$ Il s'agit d'une matière de $120 \mathrm{~h}$ par semestre ou 4,30 heures par semaine durant le semestre 6 .

$>$ Avec un coefficient 6 et un nombre de crédits 8 .

$>$ Le travail sera présenté devant un jury d'évaluation.

Le travail doit être réparti équitablement entre les membres du groupe d'étudiants

\subsubsection{Résumé des thèmes par ordre d'apparition dans le tableau 2}

\section{Thème 1}

L'étudiant doit faire une analyse fonctionnelle du profilomètre du laboratoire de métrologie de l'IOMP. Il doit ensuite établir le principe de fonctionnement et les performances métrologiques de l'appareil. A partir de là, il doit tirer des conclusions sur sont état de fonctionnement actuel et étudier les possibilités d'intervention pour son éventuelle réparation.

Thème 2

Les Normes internationales garantissent des produits et services sûrs, fiables et de bonne qualité. Pour les entreprises, elles sont des outils stratégiques permettant d'abaisser les coûts, en augmentant la productivité et en réduisant les déchets et les erreurs. Elles ouvrent l'accès à de nouveaux marchés, établissent des règles du jeu équitables pour les pays en développement et facilitent le libre-échange et le commerce équitable dans le monde. L'objectif de ce travail et de voir de plus prêt l'application des normes internationales dans les entreprises publics et privées de la wilaya de Sétif (BCR,

IRIS, AMC...) pour avoir une idée sur ce sujet et donner une assurance au consommateur local.

Tableau 2 : Liste des thèmes proposés et traités

\begin{tabular}{|l|l|}
\hline Thème & Encadreur \\
\hline $\begin{array}{l}\text { Etude fonctionnelle et } \\
\text { métrologique du profilomètre } \\
\text { mécanique avec diagnostic des } \\
\text { pannes }\end{array}$ & Dr. N. Belkhir \\
\hline $\begin{array}{l}\text { Application des normes } \\
\text { internationales dans les entreprises } \\
\text { de la wilaya de Sétif }\end{array}$ & \\
\hline $\begin{array}{l}\text { Application de la caméra CMOS à } \\
\text { l'évaluation de la vitesse des } \\
\text { particules en mouvement }\end{array}$ & Prof. L. \\
\hline $\begin{array}{l}\text { Etalonnage des micro- } \\
\text { déplacements détectés par } \\
\text { interférométrie speckle et } \\
\text { détermination de la relation } \\
\text { pixel/ } \mu \text { m }\end{array}$ & Bouamama \\
\hline $\begin{array}{l}\text { Détermination de la qualité } \\
\text { métrologique du projecteur de } \\
\text { profil et du grand microscope } \\
\text { d'atelier }\end{array}$ & Prof. L. \\
\hline $\begin{array}{l}\text { Mesure de déplacements par un } \\
\text { capteur optique }\end{array}$ & Douamama \\
\hline $\begin{array}{l}\text { Etude fonctionnelle et } \\
\text { métrologique } \\
\text { du ZKM avec diagnostic des } \\
\text { pannes }\end{array}$ & Dahgoune \\
\hline $\begin{array}{l}\text { Mesure des dimensions moyennes } \\
\text { et comparaison de deux types de } \\
\text { microparticules }\end{array}$ & Prof. L. \\
\hline $\begin{array}{l}\text { Principe de la métrologie } \\
\text { sensorielle }\end{array}$ & \\
\hline
\end{tabular}

Thème 3

L'octroi d'une caméra CMOS par l'Institut d'Optique Technique de Stuttgart qui permet de faire des acquisitions à intervalles prédéfinis, nous permet de faire des mesures de vitesse sur des particules en faibles mouvements. L'étudiant doit se familiariser à cette caméra, à son logiciel de commande et l'appliquer à 
l'évaluation de petites vitesses de mouvements de microparticules.

\section{Thème 4}

L'interférométrie speckle est une technique de métrologie optique sensible aux faibles mouvements. Comme on utilise dans ce genre de travaux des caméras de type CCD ou CMOS pour l'acquisition des images ensuite les corréler pour obtenir les franges de déplacements, donc tout se fait en pixel. Il est alors nécessaire de convertir ces déplacements en $\mu \mathrm{m}$. Pour cela, un étalonnage est nécessaire pour déterminer la relation pixel $/ \mu \mathrm{m}$ entre caméra et écran d'observation.

\section{Thème 5}

La qualité métrologique d'un système ou d'un instrument de mesure est la totalité des donnés qui caractérisent la qualité de la mesure effectuée par le système reconnu. Dans ce présent travail, on se penche sur la détermination de la qualité métrologique telles que (l'étendue de mesure, l'exactitude, la résolution, la justesse, la sensibilité la fidélité) de deux appareils de mesure existant au niveau du laboratoire pédagogique de métrologie de L'IOMP « le projecteur de profil et le grand microscope d'atelier»

\section{Thème 6}

Le fonctionnement des souris opto-mécaniques est basé sur le déplacement d'une boule encastrée dans un châssis, cette boule transmet le mouvement à deux roues crantées. Ces roues tournent entre les deux parties d'un capteur photoélectrique générant une lumière infrarouge. Selon que sa lumière infrarouge passe ou non à travers le disque, le capteur renvoie un état bas (0) ou un état haut (1). A l'aide de ces informations, l'ordinateur peut connaître la position du curseur. Dans le projet proposé, nous cherchons à enregistrer le signal de la sourie, et de le visualiser, pour qu'on puisse l'utiliser dans d'autres travaux.

\section{Thème 7}

L'étudiant doit faire une analyse fonctionnelle du ZKM du laboratoire de métrologie de l'IOMP. Il doit ensuite établir le principe de fonctionnement et les performances métrologiques de l'appareil. A partir de là, il doit tirer des conclusions sur sont état de fonctionnement actuel et étudier les possibilités d'intervention pour son éventuelle réparation.

\section{Thème 8}

Face aux exigences de la concurrence nationale et internationale, la volonté des entrepreneurs Algériens d'innover et de conquérir de nouveaux marchés, l'adhésion de l'Algérie à l'OMC, et l'ouverture du marché Algérien. La gestion et la qualité des produits comprennent plusieurs aspects, notamment la qualité organoleptique. Depuis une trentaine d'années, l'analyse et la métrologie sensorielles connaissent un développement remarquable. Ce développement revient aux préoccupations des industriels liées aussi bien à la concurrence qu'à la perception de leurs produits par les clients. L'analyse sensorielle ou métrologie sensorielle représente l'ensemble des méthodes, des outils et des instruments qui permettent d'évaluer les qualités organoleptiques d'un produit, c'est-à-dire les caractéristiques faisant intervenir les organes des sens de l'être humain : le goût, l'odorat, la vue, le toucher et l'ouïe. Elle permet de décrire et de quantifier de manière systématique l'ensemble des perceptions humaines. Globalement, l'objectif de la métrologie sensorielle est de mettre en œuvre des instruments et des méthodes visant à définir quantitativement la relation stimulus-réponse chez un sujet pour évaluer les qualités organoleptiques.

\section{Thème 9}

Les microparticules de types traceurs sont aujourd'hui beaucoup utilisées dans différents domaines telles qua la mécanique des fluides, la médecine et l'environnement. Les microparticules utilisées comme traceurs doivent avoir des caractéristiques aussi proche du fluide de transport afin qu'elles puissent tracer son mouvement avec fidélité. Dans ce travail l'étudiant doit comparer les qualités métrologiques de deux types de microparticules.

\subsection{Master "Métrologie"}

Le passage au système Licence-Master-Doctorat (LMD) représente donc une opportunité pour la formation en Master métrologie qui pourrait être reconnue à un plus haut niveau $(\mathrm{bac}+5)$ et accéder à de nouvelles passerelles avec les autres filières de formation en technologie. A ce jour, les discussions ont pris fin sur cette intégration au système LMD. Un groupe d'enseignants (entre autres Pr. L Bouamama, Dr N. Benghalem et Dr F. Roumili) ont proposé un programme assez riche pour la formation en master. Des relations étroites avec les industriels pour valider le programme ont été entretenues pour valider légalement le caractère professionnel de la formation en métrologie. Aujourd'hui basée sur des connaissances métrologiques, la formation en métrologie devrait s'appuyer davantage sur l'apprentissage et le théorique, estime le comité de préparation de la formation, qui propose alors d'articuler le programme autour de cinq situations

- Initiation des étudiants à la recherche

- Renforcement du potentiel scientifique national en bases métrologiques

- Valorisation des résultats de la recherche

- Échange scientifique à l'échelle internationale

- Promotion et diffusion des connaissances

Dans ce qui suit, nous pouvons citer les grands titres du programme prévu pour le master

- Métrologie générale et fondamentale

- Analyse statistique de données industrielles

- La normalisation technique

- Acquisition et traitement d'image 
- Mesures optiques

- Techniques microscopiques de mesure et de contrôle

Exactement en septembre 2012 le comité national de validation des nouvelles formations a émis un avis favorable à notre proposition de formation en grade de master 1et2 en métrologie selon un cahier de charge présenté. De ce fait, les étudiants qui peuvent acquérir ce grade de formation doivent attendre le début de septembre 2013 pour que l'équipe responsable de cette formation se réunisse pour se décider le nombre d'étudiants qui auront l'opportunité de poursuivre leurs études en master 1 selon les possibilités d'encadrement existantes au niveau de l'institut.

\section{Les premiers diplômés en Licence «Métrologie et contrôle industriel» Année 2012-2013}

L'institut d'optique et de mécanique de précision a eu le grand honneur de décerner et pour la première fois sur le plan national en session Juin 2013 le diplôme de Licence en métrologie et contrôle industriel exactement à dix huit étudiants avec un taux de réussite de $94.73 \%$. Le major de promotion a pu décrocher une moyenne égale à $15,83 / 20$

Les étudiants ayant obtenu leur licence peuvent postuler pour une éventuelle inscription en master 1

- L'inscription en Master est subordonnée à l'obtention d'une licence LMD

- La licence en question doit être cohérente avec l'un des Masters ouverts par notre institut.

- L'inscription se déroule en deux étapes:

\section{Etape01:Dépôt d'une demande}

Elle doit être accompagnée des documents suivants:

- Copie légalisée du diplôme Licence

- Copie du carnet de l'étudiant légalisé ou à défaut copie légalisé du relevé de notes du cursus licence.

NB : La demande doit être déposée au niveau de l'institut.

\section{Etape02:Inscription définitive :}

Après étude pédagogique et administrative des dossiers, tout candidat retenu doit procéder à son inscription définitive. Une autorisation d'inscription lui est délivrée. Le dossier comprend les pièces suivantes:

- Extrait de naissance
- Copie légalisée du relevé de notes du baccalauréat

- L'original du diplôme de la licence

- Deux photos d'identité

- Deux enveloppes timbrées libellées à l'adresse du concerné.

\section{Conclusion}

L'équipe spécialisée dans le domaine de la science de métrologie est toute heureuse d'avoir formé une promotion assez rigoureuse en métrologie. Elle a aussi le grand plaisir de contribuer avec des moyens assez simples et des motivations assez importantes pour concevoir une révolution dans ce domaine qui était dans un passé assez proche très méconnu. Cette première promotion diplômée en métrologie est à l'image de ce que nous avons mis en place. Grâce à nos formateurs de qualités et à un accompagnement optimal, leurs profils ont été particulièrement appréciés. Cette compréhension des enjeux aussi bien que de la technique font la force de ces nouveaux diplômés, qui sont armés des bons outils pour appréhender le monde du travail.

Nous souhaitons que nos étudiants peuvent mettre en place des laboratoires de mesure et d'étalonnage accrédités dans les 48 départements du territoire algériens afin d'éviter des déplacements obligatoires et qui pèsent lourd sur l'économie nationale. Enfin nous signalons que nos activités intenses sont destinées pour amener l'enseignement supérieur à répondre aux impératifs économiques et pour concevoir et dessiner un parcours organisé, lisible et progressif pour nos étudiants.

Enfin si ces premiers diplômés recevront un accueil positif sur le marché du travail nous pouvons dire que notre programme sera marqué par un succès exceptionnel.

\section{Références}

[1] N. Benghabrit-Remaoun \& Z. Rabahi-Senouci, «Le système L.M.D (Licence-Master-Doctorat) en Algérie: de l'illusion de la nécessité au choix de l'opportunité »" JHEA/RESA Vol. 7, Nos. 1\&2, 2009, pp. 189-207

[2] A. Kadri, « La construction historique du système d'enseignement supérieur en Algérie (1810-1995), in Diplômés maghrébins d'ici et d'ailleurs, Vincent Geisser, dir, Paris: CNRS.

[3] H, Khelfaoui. L'enseignement professionnel en Algérie : contraintes institutionnelles et réponses sociales, Revue: Sociologie et sociétés, Volume 40, numéro 1, 2008, p. $143-170$ 


\section{[4] www.univ-setif.dz}

[5] M. Ariba, «L’Université, ses missions et l'imposition du politique. Aperçus sur le contexte algérien d'hier et d'aujourd'hui, permanence et actualité des enjeux », Annuaire de L'Afrique du Nord, 2002-40.

[6] N. Khennafi-Benghalem, "Les perspectives et la formation en métrologie à l'ère du LMD à l'institut d'optique et de mécanique de précision à l'université Ferhat Abbas Sétif Algérie, actes d u 15eme congrès international de métrologie 3-6 octobre 2011, Paris France

[7] Agence Universitaire de la Francophonie, Les réformes de l'enseignement supérieur des pays du Maghreb et la perspective du processus de Bologne: Actes de la Rencontre des recteurs et présidents d'université des pays du Maghreb et des Conférences francophones de l'Union européenne, Marseille, 19 et 20 novembre.(2004) 